\title{
Potential utility of physical function measures to improve the risk prediction of functional disability in community-dwelling older Japanese adults: a prospective study
}

\author{
Tao Chen ${ }^{1}$, Takanori Honda ${ }^{2}$, Sanmei Chen ${ }^{3}$, Hiro Kishimoto ${ }^{4}$, Shuzo Kumagai ${ }^{5,6}$ and Kenji Narazaki ${ }^{*}$
}

\begin{abstract}
Background: While gait speed, one-leg standing balance, and handgrip strength have been shown to be independent predictors for functional disability, it is unclear whether such simple measures of physical function contribute to improved risk prediction of functional disability in older adults.
\end{abstract}

Methods: A total of 1,591 adults aged $\geq 65$ years and without functional disability at baseline were followed up for up to 7.9 years. Functional disability was identified using the database of Japan's Long-term Care Insurance System. Maximum gait speed, one-leg standing time, and handgrip strength were measured at baseline. Cox proportional hazard models were used to estimate the hazard ratios (HRs) and $95 \%$ confidence intervals ( $\mathrm{Cls}$ ) for the association of physical function and functional disability incidence. The incremental predictive value of each physical function measure for risk prediction was quantified using the difference in overall C-statistic, category-free net reclassification improvement (NRI), and integrated discrimination improvement (IDI) index.

Results: During follow-up (median: 7.8 years), functional disability was identified in 384 participants. All of the physical function measures were inversely associated with the risk of functional disability, independent of potential confounding factors. The multivariable adjusted HRs (95\% Cls) for functional disability per one standard deviation increment of maximum gait speed, one-leg-standing time, and hand grip strength were 0.73 (0.65-0.83), 0.68 (0.59-0.79), and 0.72 (0.59-0.86), respectively. Incorporation of each of maximum gait speed, one-leg-stand time, and hand grip strength into a basic model with other risk factors significantly improved C-statistic from 0.770 (95\% Cls, 0.751-0.794) to 0.778 (0.759-0.803), $0.782(0.760-0.805)$, and $0.775(0.756-0.800)$, respectively (all $p<0.05)$. A model including all three measures had the highest C-statistic of 0.787 (0.765-0.810). The improvements in risk prediction were also confirmed by category-free NRI and IDI index.

Conclusions: Adding any of the three measures to a basic model with other known risk factors significantly improved the prediction of functional disability and addition of all three measures provided further improvement of the prediction in older Japanese adults. These data provide robust evidence to support the practical utility of incorporating these simple physical function measures into functional disability risk prediction tools.

Keywords: Physical performance, Predictive value, Risk assessment, Functional limitation

\footnotetext{
* Correspondence: narazaki@fit.ac.jp

${ }^{7}$ Center for Liberal Arts, Fukuoka Institute of Technology, 3-30-1 Wajiro-

higashi, Higashi-ku, 811-0295 Fukuoka, Japan

Full list of author information is available at the end of the article
}

\section{$\triangle B M C$}

C The Author(s). 2021 Open Access This article is licensed under a Creative Commons Attribution 4.0 International License, which permits use, sharing, adaptation, distribution and reproduction in any medium or format, as long as you give appropriate credit to the original author(s) and the source, provide a link to the Creative Commons licence, and indicate if changes were made. The images or other third party material in this article are included in the article's Creative Commons licence, unless indicated otherwise in a credit line to the material. If material is not included in the article's Creative Commons licence and your intended use is not permitted by statutory regulation or exceeds the permitted use, you will need to obtain permission directly from the copyright holder. To view a copy of this licence, visit http://creativecommons.org/licenses/by/4.0/ The Creative Commons Public Domain Dedication waiver (http://creativecommons.org/publicdomain/zero/1.0/) applies to the data made available in this article, unless otherwise stated in a credit line to the data. 


\section{Introduction}

Functional disability in older adults causes increased acute care use [1], hospitalization [2], and death [3], and places considerable burdens on social, economic, and healthcare systems [4], underscoring the need for strategies to delay the onset of functional disability. Accurate identification of individuals who are at high risk is of great importance for implementing cost-effective prevention strategies. Although several multifaceted models based on self-reported risk factors have been developed for predicting incident functional disability among older adults, the discriminatory accuracy of these models ( $C$ statistic range: $0.66-0.79)$ [5-8] has only been modest [9], highlighting unexplained variation in the risk of functional disability.

Objectively measured physical functions, including gait speed, one-leg standing balance, and handgrip strength, have been shown to be strong independent predictors for future functional disability in older adults [10, 11], and have increasingly been accepted as biomarkers of aging $[12,13]$. Thus, objectively measured physical functions may contribute information beyond that obtained from other traditional risk factors and thereby improve the prediction of risk for functional disability. However, few studies have examined the added predictive values of objectively measured physical functions in the context of many other known risk factors for functional disability [14]. Furthermore, most studies have examined the predictive ability using relative risk alone, which is not sufficient to clearly define the predictive utility of a marker [15].

Given the highly dynamic nature of disability (e.g., recovery rates of activities of daily living (ADLs) disability as high as $81 \%$ within 12 months, and $65 \%$ of disability episodes lasting only 1 or 2 months) [16], prior studies with assessment intervals of $\geq 6$-month or even years [11] may underestimate the incidence of functional disability and thus lead to imprecise risk prediction. As noted in the literature [14], another common issue when studying functional disability as the outcome is loss to follow-up due to disability itself, which would underestimate the true effects [17].

To address the issues of ascertainment of functional disability and gaps in knowledge about the predictive utility of objectively measured physical functions, we linked the data from a prospective cohort study of community-dwelling older adults, including objective measures of physical function and other potential risk factors, to the Long-term Care Insurance (LTCI) system [18]. The LTCI system has been a mandatory program since it was implemented in 2000, and every adult in Japan, aged 65 years or older, is eligible for the benefits, based strictly on assessment of physical and mental disability including physicians' standardized examination
[18]. The aims of the present study were to explore whether incorporation of any of the physical function measures examined (maximum gait speed, one-leg standing balance, and handgrip strength) in a model with other common risk factors could improve prediction of functional disability, as ascertained using the LTCI system, in a nearly 8 -year prospective cohort study of older Japanese adults.

\section{Methods \\ Participants}

This prospective study used data from the Sasaguri Genkimon Study (SGS), which is an ongoing, communitybased prospective study in Sasaguri, a suburban town in Fukuoka, Japan, aiming to explore risk and protective factors related to long-term care needs [19]. Briefly, at the end of January 2011, a total of 4,979 Sasaguri residents aged $\geq 65$-years-old, and not certified as requiring long-term care according to the LTCI system, met the SGS inclusion criteria. After excluding subjects who had died or moved out of the district $(n=66)$ by the onset of the study, 4,913 subjects were invited to participate and 2,629 consented. We excluded nine subjects certified as requiring LTCI before the date of their baseline assessment, 15 with self-reported medical history of dementia or Parkinson's disease, 762 without objectively measured physical functions, and 252 with missing data regarding other risk factors. The final sample comprised 1,591 adults. Supplementary Table 1 shows the characteristics of the included participants and excluded individuals.

\section{Functional disability}

Functional disability was identified using the nationally uniform database of the LTCI system and data were provided by the municipal government office. Certification of the LTCI system has been reported in detail elsewhere [18]. Briefly, upon the request of an elderly person or their caregiver, a trained local-government official visits the home to evaluate the applicant's long-term care needs using a nationally standardized questionnaire on current physical and mental status, including paralysis and limitation of joint movement, movement and balance, complex movement, conditions requiring special assistance, conditions requiring assistance with ADLs/instrumental ADLs (IADLs), communication and cognition, and behavioral problems. A computer-based, standardized scoring system is used to calculate scores for physical and mental function and estimate the amount of time required for care in eight categories (grooming and bathing, eating, using the toilet, transferring, assistance with IADLs, behavioral problems, rehabilitation, and medical services). Finally, a local Nursing Care Needs Certification Board (comprising physicians, nurses, and other experts in health and social 
services) decides whether the older adult should be certified as requiring long-term care and assign the care needs at one of seven levels (support level, 1-2; care level, 1-5). The levels of LTCI certification have been shown to be highly correlated with the Barthel Index (Spearman's $\rho=-0.86$ ) and moderately correlated with Mini-Mental State Examination (MMSE) scores (Spearman's $\rho=-0.42$ ) [20]. We defined functional disability as the onset of long-term care needs at support level 1 or above [21, 22].

Participants were followed from the date of the baseline survey until one of the following: being ascertained as needing long-term care; death; loss to follow-up, because of moving out of town; or March 31, 2019, whichever came first. Information on death or moving out of the town was also provided by Sasaguri municipal government office using the resident registration system.

\section{Baseline physical function measures}

Physical function was assessed using three objective measures: the 5-meter maximum gait speed test, the open-eyed one-leg standing test, and the handgrip test. Although usual gait speed has been more frequently used and has been shown to have additional value in prediction of ADLs disability [14], slower maximum gait speed has also been shown to be a useful predictor of future risk of disability [23, 24]. In addition, physical challenge in maximum gait speed test may uncover earlier deficits than that using usual gait speed [25]. Therefore, maximum gait speed was selected in the present study. Details of the measurements have been reported previously [26]. Briefly, in the maximum gait speed test, the participants were asked to walk along a straight $11-\mathrm{m}$ lane as fast as possible in two trials, and the time (sec) for walking the 5-m distance between the 3-m and 8-m marks was measured in each trial using a digital stopwatch. The maximum gait speed $(\mathrm{m} / \mathrm{sec})$ was calculated by dividing $5(\mathrm{~m})$ by the shorter of the task times in the two trials. In the open-eyed one-leg standing test, the participants were asked to stand on their preferred leg for as long as possible (up to $120 \mathrm{~s}$ ) while looking at a taped mark on the wall 1-m away from the toe line. This test was performed twice, and the time (sec) to failure of the task was measured in each trial using a digital stopwatch. The longer task time in the two trials was selected as the one-leg standing time (sec). The handgrip test was performed twice for each hand using a digital grip dynamometer (TKK5401; Takei Scientific Instruments, Niigata, Japan) in a standing position. Highest value of the handgrip strength test was used in analyses. Higher values indicate better physical fitness in all of the three measures. Previous studies have shown that all three measures are highly reproducible [27-29].

\section{Other variables}

Data on age and sex were obtained from the municipality office. Living alone (yes or no), and fall experience in the previous year (yes or no) were obtained using a questionnaire. Current smoking and drinking status were assessed use questions of "do you smoke" and "do you drink" with answers of "Almost every day, Sometimes, Smoked before but not currently, Never" and "Almost every day; Sometimes, Rarely, Never", respectively. Responses of "Almost every day" and "Sometimes" were combined and classified as current smoking or drinking. Body weight and height were measured using conventional scales, and body mass index (BMI) was calculated by dividing the body weight $(\mathrm{kg})$ by height $(\mathrm{m})$ squared $\left(\mathrm{kg} / \mathrm{m}^{2}\right)$. Multimorbidity was defined as the presence of two or more of the 13 following chronic diseases: hypertension, stroke, heart disease, diabetes mellitus, hyperlipidemia, respiratory disease, digestive disease, kidney disease, osteoarthritis or rheumatism, traumatic fracture, cancer, ear disease, and eye disease. The presence of those chronic diseases was assessed using question of "Are there any chronic diseases currently being treated or any sequelae". Cognitive function was measured with the Japanese version of the MMSE. MMSE scores range from 0 to 30 with higher scores indicating better cognitive function. Cognitive impairment was defined as an MMSE score less than 24 [30]. Moderate-to-vigorous physical activity (MVPA) was objectively measured using a tri-axial accelerometer (Active style Pro HJA-350IT, Omron Healthcare, Kyoto, Japan) [31].

\section{Statistical analysis}

Baseline characteristics were described using means (standard deviation [SD]), medians (interquartile range [IQR]), or proportions with Students' t-test and chisquared test to examine differences between men and women.

The cumulative incidence of functional disability in the overall sample was plotted using Kaplan-Meier estimates. Cox proportional hazard models were used to estimate the hazard ratios (HRs) and $95 \%$ confidence intervals (CIs) for functional disability, according to sex-specific quartiles of each physical function measure. Model 1 adjusted for sex and age. Model 2 additionally adjusted for living alone, BMI, multimorbidity, fall experience in the past year, cognitive impairment, current smoker, current drinker, and MVPA. Model 3 also included all three physical function measures to examine whether the three measures were associated with functional disability independent of each. As we found no evidence of deviation from linearity examined using restricted cubic splines, HRs 
were also calculated per 1-SD increment in the respective measure. One-leg standing time was log transformed to improve the skewness for the analysis; the findings did not materially change, so results of one-leg standing time with the original scale are presented. Interactions of sex and age group $(<74$ and $\geq$ 75 years) with respective measures were also considered, to examine any potential modifying effects of sex or age.

The incremental predictive value of each physical function measure and a combination of any two measures or all three measures for risk prediction was tested by adding each measure to a basic model with other risk factors (sex, age, living alone, BMI, multimorbidity, fall experience in the past year, cognitive impairment, smoking, drinking, and MVPA). The improvement in risk discrimination was first quantified using the difference in overall C-statistic [32]. Statistical significance was based on the standard error of the differences in the C-statistic estimated from 200 bootstrap samples, using publicly available macros [33]. We further calculated the category-free net reclassification improvement (NRI) [34] and absolute integrated discrimination improvement (IDI) index [35], which have been recommended as useful supplements to the C-statistic for evaluating increment in risk prediction accuracy offered by additional markers [36]. The probabilities of incident functional disability for each model were calculated with truncation time at 7.9 years, which was the maximum follow-up. The bootstrap method with 200 replications was also used to obtain the $95 \%$ CIs.

All statistical analyses were conducted using SAS version 9.4 (SAS Institute Inc., Cary, NC). A significance level was set at two-sided $\alpha=0.05$.

\section{Results}

Over a median of 7.8 years of follow-up (IQR, 6.2 to 7.8 years), 384 participants $(24.1 \%)$ developed functional disability, 108 died prior to experiencing the event, and 62 participants were lost to follow-up ( $4 \%$ of the present sample). Cumulative incidence curve for the risk of functional disability was shown in Supplementary Fig. 1. Table 1 shows the baseline characteristics in the overall sample and by sex. The mean age (SD) at baseline was 73.3 (6.0) years and $39.8 \%$ were men. Compared to women, men were less likely to be living alone, had lower rate of fall experience, were more likely to smoke and drink, and had better physical functions but less MVPA. Participants with functional disability also had shorter MVPA time and poorer physical functions.

Table 2 shows the associations between physical function measures and incidence of functional disability. There were inverse associations across the quartiles of each physical function measure, with lower risk of incident functional disability in higher quartiles after adjusting for sex and age in model 1 ( $\mathrm{p}$ for trend $<0.0001$ ). After further adjustment in model 2, associations of all the three measures were slightly attenuated, but all remained significant. The multivariable adjusted HRs (95\% CIs) for functional disability per 1-SD increment of maximum gait speed, one-leg standing time, and hand grip strength were $0.73(0.65-0.83), 0.68(0.59-0.79)$, and $0.72(0.59-0.86)$, respectively. After mutual adjustment in model 3, associations of maximum gait speed and one-leg standing time remained significant. The association of handgrip strength was attenuated to nonsignificant when included as continuous variable. The association of all the three measures with functional disability did not vary by sex ( $p>0.20$ for all interactions).

Table 1 Baseline characteristics of the study population $(n=1,591)$

\begin{tabular}{|c|c|c|c|c|}
\hline Characteristic & Overall & Men $(n=634)$ & Women $(n=957)$ & $p$ value* \\
\hline Age, years & $73.3 \pm 6$ & $73.2 \pm 5.9$ & $73.4 \pm 6$ & 0.67 \\
\hline Living alone, \% & 13.0 & 6.3 & 17.4 & $<0.0001$ \\
\hline $\mathrm{BMI}, \mathrm{kg} / \mathrm{m}^{2}$ & $23.2 \pm 3.1$ & $23.3 \pm 2.8$ & $23.1 \pm 3.4$ & 0.16 \\
\hline Multimorbidity, \% & 47.0 & 48.4 & 46.1 & 0.36 \\
\hline Fall experience in the past year, $\%$ & 19.2 & 16.6 & 20.9 & 0.03 \\
\hline Cognitive impairment, $\%$ & 5.4 & 6.2 & 4.9 & 0.28 \\
\hline Current smoker, \% & 7.5 & 15.3 & 2.3 & $<0.0001$ \\
\hline Current drinker, \% & 39.4 & 62.2 & 24.2 & $<0.0001$ \\
\hline MVPA, min/day & $45 \pm 34.2$ & $41 \pm 31.6$ & $47.7 \pm 35.6$ & $<0.0001$ \\
\hline Maximum gait speed, $\mathrm{m} / \mathrm{sec}$ & $1.7 \pm 0.4$ & $1.8 \pm 0.4$ & $1.6 \pm 0.4$ & $<0.0001$ \\
\hline One-leg standing time, sec & $42.2(13.8-120)$ & $48.8(17.3-120)$ & $38.1(12.5-120)$ & 0.003 \\
\hline Handgrip strength, kg & $28.4 \pm 8.2$ & $36.1 \pm 6.3$ & $23.3 \pm 4.5$ & $<0.0001$ \\
\hline
\end{tabular}

Note: Continuous variables are represented as mean \pm standard deviation or median (IQR)

*Statistical significance based on chi-square tests or t-tests, as appropriate

$B M I$ body mass index; MVPA moderate-vigorous physical activity 
Table 2 Associations between objective measures of physical function and functional disability

\begin{tabular}{|c|c|c|c|c|c|c|c|c|}
\hline \multicolumn{2}{|l|}{ No. of events/subjects } & $\begin{array}{l}\text { Incidence } \\
\text { rate per } \\
1000 \\
\text { person- } \\
\text { years }\end{array}$ & \multicolumn{2}{|l|}{ Model 1} & \multicolumn{2}{|l|}{ Model 2} & \multicolumn{2}{|l|}{ Model 3} \\
\hline \multicolumn{9}{|c|}{ Maximum gait speed, $\mathrm{m} / \mathrm{sec}$} \\
\hline Q1 (lowest) & $175 / 395$ & 79.7 & 1.00 & & 1.00 & & 1.00 & \\
\hline Q2 & $97 / 391$ & 37.8 & $0.63(0.49-0.81)$ & 0.0003 & $0.66(0.51-0.86)$ & 0.002 & $0.67(0.52-0.87)$ & 0.003 \\
\hline Q3 & $75 / 404$ & 26.5 & $0.56(0.42-0.75)$ & $<0.0001$ & $0.64(0.48-0.85)$ & 0.003 & $0.70(0.52-0.94)$ & 0.02 \\
\hline Q4 (highest) & $37 / 401$ & 12.5 & $0.33(0.22-0.48)$ & $<0.0001$ & $0.40(0.27-0.59)$ & $<0.0001$ & $0.49(0.33-0.73)$ & 0.0004 \\
\hline $\mathrm{p}$ for trend & & & & $<0.0001$ & & $<0.0001$ & & 0.0008 \\
\hline Per 1 SD increment & & & $0.69(0.61-0.77)$ & $<0.0001$ & $0.73(0.65-0.83)$ & $<0.0001$ & $0.81(0.71-0.92)$ & 0.001 \\
\hline \multicolumn{9}{|c|}{ One-leg standing time, sec } \\
\hline Q1 (lowest) & $161 / 397$ & 70.5 & 1.00 & & 1.00 & & 1.00 & \\
\hline Q2 & $131 / 398$ & 52.3 & $1.05(0.83-1.34)$ & 0.67 & $1.06(0.84-1.36)$ & 0.62 & $1.16(0.91-1.49)$ & 0.22 \\
\hline Q3 & $55 / 318$ & 24.6 & $0.60(0.43-0.83)$ & 0.002 & $0.67(0.49-0.93)$ & 0.02 & $0.78(0.56-1.09)$ & 0.14 \\
\hline Q4 (highest) & $37 / 478$ & 10.5 & $0.36(0.24-0.53)$ & $<0.0001$ & $0.41(0.28-0.61)$ & $<0.0001$ & $0.51(0.34-0.77)$ & 0.001 \\
\hline$p$ for trend & & & & $<0.0001$ & & $<0.0001$ & & 0.001 \\
\hline Per 1 SD increment & & & $0.64(0.55-0.73)$ & $<0.0001$ & $0.68(0.59-0.79)$ & $<0.0001$ & $0.74(0.63-0.86)$ & $<0.0001$ \\
\hline \multicolumn{9}{|l|}{ Handgrip strength, kg } \\
\hline Q1 (lowest) & $149 / 377$ & 69.0 & 1.00 & & 1.00 & & 1.00 & \\
\hline Q2 & $116 / 359$ & 50.5 & $0.94(0.74-1.21)$ & 0.63 & $1.02(0.79-1.30)$ & 0.89 & $1.13(0.88-1.46)$ & 0.33 \\
\hline Q3 & $71 / 393$ & 26.3 & $0.63(0.47-0.85)$ & 0.0024 & $0.73(0.54-0.98)$ & 0.04 & $0.85(0.62-1.15)$ & 0.29 \\
\hline Q4 (highest) & $48 / 462$ & 14.2 & $0.45(0.31-0.64)$ & $<0.0001$ & $0.53(0.37-0.76)$ & 0.0005 & $0.69(0.48-0.997)$ & 0.048 \\
\hline$p$ for trend & & & & $<0.0001$ & & 0.0003 & & 0.047 \\
\hline Per 1 SD increment & & & $0.64(0.53-0.76)$ & $<0.0001$ & $0.72(0.59-0.86)$ & 0.0005 & $0.83(0.68-1.01)$ & 0.07 \\
\hline
\end{tabular}

Note: Model 1 is adjusted for sex and age

Model 2 is adjusted for sex, age, living alone, body mass index, multimorbidity, fall experience in the past year, cognitive impairment, smoking, drinking, and moderate-to-vigorous physical activity

Model 3 is adjusted for variables in model 2 plus the other physical function measures

The sex-specific quartile cut points were: gait speed, 1.6, 1.8, and $2.1 \mathrm{~m} / \mathrm{sec}$ for men, and 1.4, 1.6, and $1.9 \mathrm{~m} / \mathrm{sec}$ for women; one-leg standing time, $17.3,48.8$, and $120 \mathrm{~s}$ for men, and $12.5,38.1$, and $120 \mathrm{~s}$ for women; handgrip strength, 32.0, 36.0, and $40.0 \mathrm{~kg}$ for men, and 20.5, 23.0, and 26.0 kg for women

HRs hazard ratios; Cls confidence intervals

As shown in Supplementary Tables 2, the associations were in the same direction in both sexes. There was also no evidence of age interaction $(p>0.05)$. Thus, in the subsequent analyses, overall sample was used to maximize the power.

Maximum gait speed, one-leg standing time, and handgrip strength improved the C-statistic of the basic model from 0.770 (95\% CIs, $0.751-0.794$ ) to 0.778 (0.759-0.803), $0.782(0.760-0.805)$, and 0.775 (0.7560.800 ), respectively (all $p<0.05$ ) (Table 3 ). An addition of any combination of two measures also significantly improved the C-statistic of the basic model (all $p<0.05$ ). A model including all three measures produced the highest C-statistic (0.787), when compared to the basic model or a model including only one physical function measure (all $p<0.05$ ). The category-free NRI and IDI index also confirmed the improvements in risk prediction.
Similar results were observed when adding quartiles of physical function measure to the basic model (Supplementary Table 3).

\section{Discussion}

In this nearly 8 -year prospective study of communitydwelling older Japanese adults, the incorporation of each of the three physical functions (maximum gait speed, one-leg standing time, and handgrip strength) to a model with well-known risk factors improved discrimination of future functional disability and the simultaneous addition of all three measures further improved the risk prediction. These results provide strong evidence to support the practical utility of incorporating these simple physical function measures into functional disability risk prediction tools.

Our findings of associations between physical functions and risk of functional disability are generally 
Table 3 The improvement in functional disability risk discrimination when adding each physical function measure as continuous variables to the basic model

\begin{tabular}{llll}
\hline & C-statistic & Category-free NRI & Absolute IDI \\
\hline Basic model & $0.770(0.751$ to 0.794$)$ & Reference & Reference \\
Basic model + maximum gait speed & $0.778(0.759 \text { to } 0.803)^{*}$ & $0.267(0.173 \text { to } 0.368)^{*}$ & $0.015(0.007 \text { to } 0.024)^{*}$ \\
Basic model + one-leg standing time & $0.782(0.760 \text { to } 0.805)^{*}$ & $0.405(0.293 \text { to } 0.516)^{*}$ & $0.022(0.017 \text { to } 0.028)^{*}$ \\
Basic model + handgrip strength & $0.775(0.756 \text { to } 0.800)^{*}$ & $0.129(0.013 \text { to } 0.242)^{*}$ & $0.009(0.004 \text { to } 0.015)^{*}$ \\
Basic model + maximum gait speed + one-leg standing time & $0.785(0.764 \text { to } 0.809)^{*}$ & $0.448(0.336 \text { to } 0.551)^{*}$ & $0.031(0.023 \text { to } 0.041)^{*}$ \\
Basic model + maximum gait speed + handgrip strength & $0.781(0.761 \text { to } 0.805)^{*}$ & $0.308(0.196 \text { to } 0.427)^{*}$ & $0.019(0.010 \text { to } 0.029)^{*}$ \\
Basic model + one-leg standing time + handgrip strength & $0.784(0.763 \text { to } 0.807)^{*}$ & $0.378(0.272 \text { to } 0.517)^{*}$ & $0.027(0.021 \text { to } 0.035)^{*}$ \\
Basic model + all three physical function measures & $0.787(0.765 \text { to } 0.810)^{*}$ & $0.429(0.322 \text { to } 0.554)^{*}$ & $0.034(0.025 \text { to } 0.045)^{*}$ \\
\hline
\end{tabular}

Note: Basic model: sex, age, living alone, body mass index, multimorbidity, fall experience in the past year, cognitive impairment, smoking, drinking, and moderate-to-vigorous physical activity

${ }^{*} p<0.05$ for difference with the basic model

$N R I$ net reclassification improvement; IDI integrated discrimination improvement

consistent with a limited number of Japanese cohort studies of usual or maximum gait speed [24, 37, 38], one-leg standing time [39], and handgrip strength [37, 40], which assessed functional disability using the LTCI system. Although no significant association of one-leg standing time [24] or handgrip strength [39] with functional disability was reported, the relatively small sample sizes $(n=60$ to 784$)$ and short follow-up periods (5-6 years) may have limited the statistical power in these two earlier studies. A recent meta-analysis also revealed that poorer performance in gait speed, one-leg standing balance, and handgrip strength, measured at baseline, were associated with higher odds of disability in ADLs or instrumental ADLs at follow-up [10]. Our findings also confirmed the inverse association between each of these physical function measures and the risk of functional disability as ascertained using the LTCI system.

Although extensive research has shown associations between objective physical function measures and functional disability, whether such measures have practical utility has been unclear because a significant association does not necessarily translate into predictive utility [15]. A recent study using a pooled analysis of 27,200 older adults with disability defined by selected tasks (bathing or dressing, and mobility difficulty) reported that usual gait speed significantly increased the area under the receiver operator characteristic curve (C-statistic), after accounting for age, sex, BMI, prior hospitalization, and selected chronic conditions over 3 years of follow-up [14]. A key finding of the present study, which is the first to report it, is that adding each of one-leg standing time and handgrip strength, in addition to maximum gait speed, significantly improved the C-statistic of a basic model with well-known risk factors for functional disability. The NRI and IDI results in the present study also confirmed the improved risk discrimination. These findings extend prior observations on the associations of physical function measures with risk of functional disability, by providing novel evidence that incorporating each of these physical function measures has the potential to provide a meaningful improvement in identification of older adults at risk of future functional disability. One possible explanation for these findings is that objective measures of physical functions, acting as biomarker of aging [12, 13], provide informative indications of subclinical disease and underlying aging processes, which may not be captured by other traditional risk factors.

In addition, we also found incorporation of all three physical function measures further improved the risk prediction of functional disability, suggesting each physical function measure may capture specific underlying characteristics that is of additional value in improving the risk prediction of functional disability. The independent associations, particularly of maximum gait speed and one-leg standing time, observed in the present study also support that different measures of physical function may reflect distinct abilities. These findings provided the rationale of a combination use of different physical function measures in assessing the risk of functional disability. Given that the physical function measures included in the present study are all highly reproducible tests [27-29] and could be done quickly in different settings, including these simple objective measures of physical function as part of the routine geriatric assessment could be practical and useful for identification of high-risk individuals.

The strengths of the present study include the large community-based population and the use of the nationally standardized LTCI database, which allowed us to conduct a more formal time-to-disability analysis with a follow-up rate of almost $100 \%$. However, the present study also had several limitations. First, because an older person must contact the municipal government to have 
the care needs officially certified [18], some individuals with disability may have failed to report. Thus, the present study may have underestimated the incidence of functional disability and the true associations of physical function measures. Second, although a range of important risk factors were included in the analysis, unmeasured confounding or residual effects may still exist. Third, a large proportion of participants were excluded in the present study, mainly owing to missing data. Included participants were younger, had lower rates of falls and cognitive impairment, were less likely to smoke or drink, and to have poorer performance in the maximum gait speed and one-leg standing tests, although the participants had a higher rate of multimorbidity than did those excluded from the present study (Supplementary Table 1). Thus, participants in the present study could have been physically healthier than the general population, and it is possible that the observed results have underestimated the strength of the association between physical function measures and functional disability. In addition, the generalizability of the findings may also be limited because the present study was undertaken in a single Japanese town.

In summary, the present study demonstrated that maximum gait speed, one-leg standing time, and handgrip strength have a predictive value for functional disability beyond known risk factors and a combination use of the three measures further improve the risk prediction in community-dwelling, older Japanese adults. These findings highlight the predictive utility of incorporating these simple measures of physical function into the screening setting, to identify older adults who are at high risk of developing functional disability.

\section{Abbreviations}

ADLs: Activities of daily living; BMl: Body mass index; Cls: Confidence intervals; HRs: Hazard ratios; LTCl: Long-term Care Insurance; MMSE: MiniMental State Examination; MVPA: Moderate-to-vigorous physical activity; NRI: Category-free net reclassification improvement; IDI: Integrated discrimination improvement; SD: Standard deviation; IQR: Interquartile rang; SGS: Sasaguri Genkimon Study

\section{Supplementary Information}

The online version contains supplementary material available at https:/doi. org/10.1186/s12877-021-02415-3.

\section{Additional file 1:}

Additional file 2 .

\section{Acknowledgements}

We would like to thank Ms. Yuka Haeuchi, Dr. Yu Nofuji, Ms. Eri Shiokawa, and the municipal staff in the primary care-giving division in Sasaguri, who helped us coordinate the survey in the community.

\section{Authors' contributions}

Conception and design: TC and KN. Acquisition of data: TC, TH, SC, SK and KN. Analysis and interpretation of data: TC. Drafting the manuscript: TC.
Critically revising manuscript: TH, SC, HK, SK and KN. All authors approved the submitted version.

\section{Funding}

This work was partly supported by a Health and Labour Sciences Research Grant of the Ministry of Health, Labour and Welfare of Japan (2013-

Ninchisho-Ippan-004) to SK, a research grant from the Mitsui Sumitomo Insurance Welfare Foundation to SC, a grant from Sasaguri Town to SK (2011-2016), and Japan Society for the Promotion of Science KAKENHI Grant Number JP17K09146 and JP20H04030 to KN.

Availability of data and materials

The datasets used and/or analyzed during the current study are available from the corresponding author on reasonable request.

\section{Declarations}

Ethics approval and consent to participate

Written informed consent was obtained from all subjects. This study was conducted in accordance with the Declaration of Helsinki and approved by the Institutional Review Board of the Institute of Health Science, Kyushu University.

\section{Consent for publication}

NA

\section{Competing interests}

All authors report no conflicts of interest.

\section{Sponsor's Role}

None of the funding sources had any role in the study design, data analysis, data interpretation, writing of the manuscript, or decision about submission.

\section{Author details}

'Sport and Health Research Center, Department of Physical Education, Tongji University, 1239 Siping Road, 200092 Shanghai, China. ${ }^{2}$ Department of Epidemiology and Public Health, Graduate School of Medical Sciences, Kyushu University, 3-1-1 Maidashi, Higashi-ku, 812-8582 Fukuoka, Japan. ${ }^{3}$ Department of Global Health Nursing, Graduate School of Biomedical and Health Sciences, Hiroshima University, 1-2-3 Kasumi, Minami Ward, 734-8553 Hiroshima, Japan. ${ }^{4}$ Faculty of Arts and Science, Kyushu University, 744 Motooka Nishi-ku, 819-0395 Fukuoka, Japan. ${ }^{5}$ Institute of Convergence Bio-Health, Dong-A University, 37 Nakdong-daero 550 beon-gil, Hadan-dong, Saha-gu, 49-315 Busan, South Korea. ${ }^{6}$ Kumagai Institute of Health Policy, 4-47-1 Hiratadai, 816-0812 Kasuga-shi, Fukuoka, Japan. ${ }^{7}$ Center for Liberal Arts, Fukuoka Institute of Technology, 3-30-1 Wajiro- higashi, Higashi-ku, 811-0295 Fukuoka, Japan.

Received: 24 October 2020 Accepted: 10 August 2021

Published online: 01 September 2021

\section{References}

1. Wu CY, Hu HY, Li CP, et al. The association between functional disability and acute care utilization among the elderly in taiwan. Arch Gerontol Geriatr. 2013;57:177-183. doi: https://doi.org/10.1016/j.archger.2013.04.011

2. Mor V, Wilcox $V$, Rakowski W, et al. Functional transitions among the elderly: Patterns, predictors, and related hospital use. Am J Public Health. 1994;84: 1274-1280. doi: https://doi.org/10.2105/ajph.84.8.1274

3. Marengoni A, von Strauss E, Rizzuto D, et al. The impact of chronic multimorbidity and disability on functional decline and survival in elderly persons. A community-based, longitudinal study. J Intern Med. 2009;265: 288-295. doi: https://doi.org/10.1111/j.1365-2796.2008.02017.x

4. World Health Organization. World report on ageing and health. Geneva: World Health Organization; 2015. https://apps.who.int/iris/bitstream/ha ndle/10665/186463/9789240694811_eng.pdf?sequence=1. Accessed 12 Aug 2020

5. Covinsky KE, Hilton J, Lindquist K, et al. Development and validation of an index to predict activity of daily living dependence in community-dwelling elders. Med Care. 2006:44:149-157. doi: https://doi.org/10.1097/01.mlr.00001 96955.99704.64 
6. Clark DO, Stump TE, Tu W, et al. A comparison and cross-validation of models to predict basic activity of daily living dependency in older adults. Med Care. 2012;50:534-539. doi: https://doi.org/10.1097/MLR.0b013e318245a $50 \mathrm{c}$

7. Kim DH, Newman AB, Lipsitz LA. Prediction of severe, persistent activity-ofdaily-living disability in older adults. Am J Epidemiol. 2013;178:1085-1093. doi: https://doi.org/10.1093/aje/kwt097

8. Nüesch E, Pablo P, Dale CE, et al. Incident disability in older adults: Prediction models based on two british prospective cohort studies. Age Ageing. 2015;44:275-282. doi: https://doi.org/10.1093/ageing/afu159

9. Ohman EM, Granger CB, Harrington RA, et al. Risk stratification and therapeutic decision making in acute coronary syndromes. Jama. 2000;284 876-878. doi: https://doi.org/10.1001/jama.284.7.876

10. Wang DXM, Yao J, Zirek Y, et al. Muscle mass, strength, and physical performance predicting activities of daily living: A meta-analysis. J Cachexia Sarcopenia Muscle. 2020;11:3-25. doi: https://doi.org/10.1002/jcsm.12502

11. Cavanaugh EJ, Richardson J, McCallum CA, et al. The predictive validity of physical performance measures in determining markers of preclinical disability in community-dwelling middle-aged and older adults: A systematic review. Phys Ther. 2018;98:1010-1021. doi: https://doi.org/10.1 093/ptj/pzy109

12. Lara J, Cooper R, Nissan J, et al. A proposed panel of biomarkers of healthy ageing. BMC Med. 2015;13:222. doi: https://doi.org/10.1186/s12916-015-04 70-9

13. Justice JN, Cesari M, Seals DR, et al. Comparative approaches to understanding the relation between aging and physical function. J Gerontol A Biol Sci Med Sci. 2016;71:1243-1253. doi: https://doi.org/10.1093/gerona/ glv035

14. Perera S, Patel KV, Rosano C, et al. Gait speed predicts incident disability: A pooled analysis. J Gerontol A Biol Sci Med Sci. 2016;71:63-71. doi: https:// doi.org/10.1093/gerona/glv126

15. Pepe $M S$, Janes $H$, Longton $G$, et al. Limitations of the odds ratio in gauging the performance of a diagnostic, prognostic, or screening marker. Am J Epidemiol. 2004;159:882-890. doi: https://doi.org/10.1093/aje/kwh101

16. Hardy SE, Gill TM. Recovery from disability among community-dwelling older persons. Jama. 2004;291:1596-1602. doi: https://doi.org/10.1001/jama.2 91.13.1596

17. Hardy SE, Allore H, Studenski SA. Missing data: A special challenge in aging research. J Am Geriatr Soc. 2009;57:722-729. doi: https://doi.org/10.1111/j.1 532-5415.2008.02168.x

18. Tsutsui T, Muramatsu N. Care-needs certification in the long-term care insurance system of japan. J Am Geriatr Soc. 2005;53:522-527. doi: https:// doi.org/10.1111/j.1532-5415.2005.53175.x

19. Narazaki K, Nofuji $Y$, Honda T, et al. Normative data for the montreal cognitive assessment in a japanese community-dwelling older population. Neuroepidemiology. 2013;40:23-29. doi: https://doi.org/10.1159/000339753

20. Arai $Y$, Zarit $\mathrm{SH}$, Kumamoto $\mathrm{K}$, et al. Are there inequities in the assessment of dementia under japan's Itc insurance system? Int J Geriatr Psychiatry. 2003;18:346-352. doi: https://doi.org/10.1002/gps.836

21. Komiyama T, Ohi T, Miyoshi Y, et al. Association between tooth loss, receipt of dental care, and functional disability in an elderly japanese population: The tsurugaya project. J Am Geriatr Soc. 2016;64:2495-2502. doi: https://doi. org/10.1111/jgs.14390

22. Chen T, Honda T, Chen S, et al. Dose-response association between accelerometer-assessed physical activity and incidence of functional disability in older japanese adults: A 6-year prospective study. J Gerontol A Biol Sci Med Sci. 2020. doi: https://doi.org/10.1093/gerona/glaa046

23. Rosso AL, Metti AL, Faulkner K, et al. Associations of usual pace and complex task gait speeds with incident mobility disability. J Am Geriatr Soc. 2019;67:2072-2076. doi: https://doi.org/10.1111/jgs.16049

24. Tainaka K, Takizawa T, Katamoto S, et al. Six-year prospective study of physical fitness and incidence of disability among community-dwelling japanese elderly women. Geriatr Gerontol Int. 2009;9:21-28. doi: https://doi. org/10.1111/j.1447-0594.2008.00492.x

25. Rosso AL, Metti AL, Faulkner K, et al. Complex walking tasks and risk for cognitive decline in high functioning older adults. J Alzheimers Dis. 2019;71: S65-s73. doi: https://doi.org/10.3233/jad-181140

26. Narazaki K, Matsuo E, Honda T, et al. Physical fitness measures as potential markers of low cognitive function in japanese community-dwelling older adults without apparent cognitive problems. J Sports Sci Med. 2014;13:590596.
27. Steffen TM, Hacker TA, Mollinger L. Age- and gender-related test performance in community-dwelling elderly people: Six-minute walk test, berg balance scale, timed up \& go test, and gait speeds. Phys Ther. 2002;82: 128-137. doi: https://doi.org/10.1093/ptj/82.2.128

28. Franchignoni F, Tesio L, Martino MT, et al. Reliability of four simple, quantitative tests of balance and mobility in healthy elderly females. Aging (Milano). 1998;10:26-31. doi: https://doi.org/10.1007/bf03339630

29. Roberts HC, Denison HJ, Martin HJ, et al. A review of the measurement of grip strength in clinical and epidemiological studies: Towards a standardised approach. Age Ageing. 2011;40:423-429. doi: https://doi.org/1 0.1093/ageing/afr051

30. Ideno Y, Takayama M, Hayashi K, et al. Evaluation of a japanese version of the mini-mental state examination in elderly persons. Geriatr Gerontol Int. 2012;12:310-316. doi: https://doi.org/10.1111/j.1447-0594.2011.00772.x

31. Chen T, Narazaki K, Honda T, et al. Tri-axial accelerometer-determined daily physical activity and sedentary behavior of suburban community-dwelling older japanese adults. J Sports Sci Med. 2015;14:507-514.

32. Pencina MJ, D'Agostino RB. Overall $\mathrm{c}$ as a measure of discrimination in survival analysis: Model specific population value and confidence interval estimation. Stat Med. 2004;23:2109-2123. doi: https://doi.org/10.1002/sim.1 802

33. Cook NR. Risk prediction modeling: Division of preventive medicine (Brigham \& Women's Hospital). Available at: https://ncook.bwh.harvard.edu/ sas-macros.html. Accessed 28 Apr 2020.

34. Pencina MJ, D'Agostino RB, Sr., Steyerberg EW. Extensions of net reclassification improvement calculations to measure usefulness of new biomarkers. Stat Med. 2011;30:11-21. doi: https://doi.org/10.1002/sim.4085

35. Pencina MJ, D'Agostino RB, Sr., D'Agostino RB, Jr., et al. Evaluating the added predictive ability of a new marker: From area under the roc curve to reclassification and beyond. Stat Med. 2008;27:157-172; discussion 207 112. doi: https://doi.org/10.1002/sim.2929

36. Pencina MJ, D'Agostino RB, Sr., Demler OV. Novel metrics for evaluating improvement in discrimination: Net reclassification and integrated discrimination improvement for normal variables and nested models. Stat Med. 2012;31:101-113. doi: https://doi.org/10.1002/sim.4348

37. Makizako $H$, Shimada $H$, Doi T, et al. Impact of physical frailty on disability in community-dwelling older adults: A prospective cohort study. BMJ Open. 2015;5:e008462. doi: https://doi.org/10.1136/bmjopen-2015-008462

38. Abe T, Kitamura A, Taniguchi $Y$, et al. Pathway from gait speed to incidence of disability and mortality in older adults: A mediating role of physical activity. Maturitas. 2019;123:32-36. doi: https://doi.org/10.1016/j.maturitas.2 019.02.002

39. Moriya S, Murata A, Kimura S, et al. Predictors of eligibility for long-term care funding for older people in japan. Australas J Ageing. 2013;32:79-85. doi: https://doi.org/10.1111/j.1741-6612.2012.00601.x

40. Otsuka H, Kobayashi $H$, Suzuki K, et al. Mobility performance among healthy older adults eligible for long-term care in japan: A prospective observational study. Aging Clin Exp Res. 2019. doi: https://doi.org/10.1007/s40520-019-014 04-2

\section{Publisher's Note}

Springer Nature remains neutral with regard to jurisdictional claims in published maps and institutional affiliations.

Ready to submit your research? Choose BMC and benefit from:

- fast, convenient online submission

- thorough peer review by experienced researchers in your field

- rapid publication on acceptance

- support for research data, including large and complex data types

- gold Open Access which fosters wider collaboration and increased citations

- maximum visibility for your research: over $100 \mathrm{M}$ website views per year

At $\mathrm{BMC}$, research is always in progress.

Learn more biomedcentral.com/submissions 